\title{
AN INVARIANT FOR MODULES OVER A DISCRETE VALUATION RING ${ }^{1}$
}

\author{
R. O. STANTON
}

ABSTRACT. Warfield has recently defined a new class of invariants for mixed modules over a discrete valuation ring. These invariants, along with the Ulm invariants, enable Warfield to prove an analogue to Ulm's theorem. Warfield's definition contains two shortcomings. The invariants are defined for a limited class of modules. Moreover it is difficult to show that the invariants are well defined. This paper defines a new invariant which coincides with that of Warfield, and overcomes both difficulties.

1. Preliminaries. $R$ will be a discrete valuation ring throughout, and $p$ will represent a generator of its maximal ideal. If $M$ is an $R$-module and $S$ is a subset of $M,[S]$ represents the submodule generated by $S$. The symbol $\bigoplus_{i \in I} M_{i}$ denotes the direct sum of the $R$-modules $M_{i}(i \in I)$. The dimension of the vector space $V$ over the field $R / p R$ is written $d(V)$.

If $M$ is an $R$-module and $\alpha$ is an ordinal, $p^{\alpha} M$ is defined inductively: $p^{\alpha+1} M=p\left(p^{\alpha} M\right)$ and, for a limit ordinal $\alpha, p^{\alpha} M=\bigcap_{\beta<\alpha} p^{\alpha} M$. Given $m \in M$, the height $h(m)$ of $m$ is $\alpha$ if $m \in p^{\alpha} M \backslash p^{\alpha+1} M$. If $m \in p^{\alpha} M$ for all $\alpha, h(m)=$ $\infty$. Let $\mu=\left\{a_{0}, \alpha_{1}, \ldots\right\}$ be a sequence in which each $\alpha_{i}$ is either an ordinal or the symbol $\infty_{.} \mu$ is a height sequence when (1) if $\alpha_{i}=\infty$, then $\alpha_{i+1}=\infty$, and (2) if $\alpha_{i}$ is an ordinal, either $\alpha_{i+1}=\infty$ or $\alpha_{i+1}$ is an ordinal such that $\alpha_{i+1}>\alpha_{i}$. The height sequences $\mu$ and $\nu=\left\{\beta_{0}, \beta_{1}, \ldots\right\}$ are equivalent if there are integers $m$ and $n$ such that $\alpha_{i+m}=\beta_{i+n}$, for all $i \geq 0$. If $M$ is an $R$-module and $x \in M$, the height sequence $H(x)$ of $x$ is the sequence $\left\{h\left(p^{i} x\right)\right\}, 0 \leq i<\infty$.

The $R$-module $M$ is of torsion free rank one if, for any two elements $x$ and $y$ of infinite order in $M$, there are nonzero elements $r$ and $s$ in $R$ such that $r x=s y$. If $M$ is of torsion free rank one, then it is clear that any two

Received by the editors March 25, 1974 .

AMS (MOS) subject classifications (1970). Primary 13C05, 20K25; Secondary $13 \mathrm{H} 99$.

Key words and phrases. Discrete valuation ring, module of torsion free rank one, height sequence.

1 This article is taken from the author's doctoral dissertation, which was written under the direction of Dr. Elbert A. Walker. 
elements of infinite order have equivalent height sequences. This equivalence class of height sequences is thus an invariant $H(M)$ of $M$. If $M=$ $\bigoplus_{i \in I} M_{i}$, where each $M_{i}$ has torsion free rank one, and $e$ is an equivalence class of height sequences, $g(e, M)$ is defined to be the cardinal number of summands $M_{i}$ such that $H\left(M_{i}\right)=e$. In [1], Warfield has shown that $g(e, M)$ does not depend on the choice of the decomposition of $M$, so $g(e, M)$ is an invariant of $M$. Moreover, Warfield has extended the definition to direct summands of $M$. In this paper, an invariant will be defined for all $R$-modules which will coincide with $g(e, M)$ when the latter is defined.

2. An invariant for modules over a discrete valuation ring. If $M$ is a reduced module and $\mu=\left\{\alpha_{0}, \alpha_{1}, \ldots\right\}$ is a height sequence, with $a_{n} \neq \infty$ for all $n$, define:

$$
\begin{aligned}
\mu M & =\left\{m \in M: h\left(p^{i} m\right) \geq \alpha_{i}, i=0,1,2, \ldots\right\}, \text { and } \\
\mu^{*} M & =\left[\left\{m \in \mu M: \text { for infinitely many } i, h\left(p^{i} m\right)>\alpha_{i}\right\}\right] .
\end{aligned}
$$

$\mu M$ and $\mu^{*} M$ are both submodules of $M$, and $\mu M / \mu^{*} M$ is a vector space over $R / p R$.

If $\mu=\left\{\alpha_{0}, \alpha_{1}, \ldots\right\}$ is a height sequence and $i \geq 0$, the height sequence $\mu_{i}$ is defined by $\mu_{i}=\left\{a_{i}, a_{i+1}, \ldots\right\}$. Clearly $\mu$ and $\mu_{i}$ belong to the same equivalence class of height sequences.

Lemma 1. The map $\phi_{i}: \mu M / \mu^{*} M \rightarrow \mu_{i} M / \mu_{i}^{*} M$, defined by $\phi_{i}\left(x+\mu^{*} M\right)=$ $p^{i} x+\mu_{i}^{*} M$, is a monomorphism.

Proof. Since $x \in \mu^{*} M$ implies $p^{i} x \in \mu_{i}^{*} M, \phi_{i}$ is well defined.

To show $\phi_{i}$ is monic, it suffices to show $\phi_{1}$ is monic. For then $\phi_{i}$ is a composition of monics. Let $x+\mu^{*} M \in \operatorname{Ker} \phi_{1}$. Then $p x=\Sigma_{t=1}^{n}{ }_{t}{ }_{t}{ }_{t}$, where each $x_{t}$ is in the generating set defining $\mu_{1}^{*} M$. Let $\left\{\beta_{0}^{t}, \beta_{1}^{t}, \ldots\right\}$ be the height sequence of $x_{t}$. Then $\beta_{k}^{t} \geq \alpha_{k+1}$ for all $k$, and for each $t$ there are infinitely many $k$ for which $\beta_{k}^{t}>\alpha_{k+1}$. We may write $x_{t}=p y_{t}$, where $y_{t}$ has height sequence $\left\{\gamma^{t}, \beta_{0}^{t}, \beta_{1}^{t}, \ldots\right\}$, with $\gamma^{t} \geq a_{0}$. Thus $y_{t} \in \mu^{*} M$ and $p\left(x-\Sigma r_{t} y_{t}\right)=0$. $x-\Sigma r_{t} y_{t}$ is a torsion element of $\mu M$, hence is in $\mu^{*} M$. Thus $x=\left(x-\Sigma r_{t} y_{t}\right)+\Sigma r_{t} y_{t}$ is an element of $\mu^{*} M$, so $\phi_{1}$ is monic.

Corollary 2. If $i \leq j$, then $d\left(\mu_{i} M / \mu_{i}^{*} M\right) \leq d\left(\mu_{j} M / \mu_{j}^{*} M\right)$.

As a consequence of Corollary 2, if $\mu$ and $\nu$ are two representatives of the same equivalence class of height sequences, then $\lim _{i \rightarrow \infty} d\left(\mu_{i} M / \mu_{i}^{*} M\right)=$ 
$\lim _{j \rightarrow \infty} d\left(\nu_{i} M / \nu_{i}^{*} M\right)$. If $e$ is an equivalence class of height sequences and $\mu$ is a representative of $e$, the cardinal number $S(e, M)=\lim _{i \rightarrow \infty} d\left(\mu_{i} M / \mu_{i}^{*} M\right)$ is well defined. $S(e, M)$ is an invariant of $M$.

Let the reduced module $M$ have torsion free rank one and $\mu=\left\{a_{0}, a_{1}, \ldots\right\}$ be a representative of the equivalence class of height sequences determined by $M$. If $x \in \mu^{*} M$ has height sequence $\left\{\beta_{0}, \beta_{1}, \ldots\right\}$, then $\beta_{i}>\alpha_{i}$ for all but a finite number of $i$. In particular, no elements of $\mu^{*} M$ have height sequence $\mu$.

Theorem 3. Let $M$ be a reduced module of torsion free rank one. If $e=$ $H(M)$, then $S(e, M)=1$; if $f \neq H(M)$, then $S(f, M)=0$.

Proof. Let $x$ be an element of $M$ of infinite order and height sequence $\mu \in e$. It will be shown that any nonzero coset of $\mu M / \mu^{*} M$ has a representative of the form $a x+\mu^{*} M$, where $a$ is a unit of $R$. Let $y \in \mu M$, and let $\nu$ be the height sequence of $y$. If $y \notin \mu^{*} M$, there is $i \geq 0$ such that $\nu_{i}=\mu_{i}$. Since $M$ has torsion free rank one, there is a unit $a$ and $m \geq 0$ such that $p^{m} a x=$ $p^{m} y$. Now $y-a x \in \mu^{*} M$, so $y+\mu^{*} M=a x+\mu^{*} M$.

Let $\sigma \in f$ and let $z \in \sigma M \backslash \sigma^{*} M$. Then all but a finite number of ordinals in the height sequence of $z$ coincide with $\sigma$. This implies $H(z)$ is equivalent to $\sigma$, a contradiction.

Lemma 4. Let $M=\bigoplus_{I} M_{i}$ be a direct sum of reduced modules. Then $\mu M / \mu^{*} M \simeq \bigoplus_{I} \mu M_{i} / \mu^{*} M_{i}$

Proof. For each $x \in M$, write $x=\Sigma_{I} x_{i}$, where each $x_{i} \in M_{i^{*}}$. Define $\phi: \mu M / \mu^{*} M \rightarrow \bigoplus_{I} \mu M_{i} / \mu^{*} M_{i}$ by $\phi\left(x+\mu^{*} M\right)=\Sigma_{I}\left(x_{i}+\mu^{*} M_{i}\right)$. It is routine to prove $\phi$ is an isomorphism.

Theorem 5. Let $M=\bigoplus_{I} M_{i}$ be a direct sum of reduced modules of torsion free rank one. Then, for any equivalence class $e$ of height sequences, $S(e, M)$ is the number of summands $M_{i}$ whose equivalence class of height sequences is $e$.

Proof. This is a consequence of Theorem 3 and Lemma 4.

Let $e$ be the class containing $\infty$ and $M$ be a module. Then $S(e, M)$ is defined to be the dimension of the divisible part of $M / T$ as a vector space over the quotient field of $R$, where $T$ is the torsion part of $M$.

This completes the task of generalizing Warfield's invariant $g(e, M)$. 
Theorem 6. Let $M$ be a summand of a direct sum of modules of torsion free rank one. Then $S(e, M)=g(e, M)$, for any equivalence class $e$ of height sequences.

\section{REFERENCE}

1. R. B. Warfield, Jr., Classification theorems for p-groups and modules over a discrete valuation ring, Bull. Amer. Math. Soc. 78 (1972), 88-92. MR 45 \#378.

DEPARTMENT OF MATHEMATICS, KANSAS STATE UNIVERSITY, NANHATTAN, KANSAS 66506

Current address: Department of Mathematics, Benedict College, Columbia, South Carolina 29204

PROCEEDINGS OF THE

AMERICAN MATHEMATICAL SOCIETY

Volume 49, Number 1, May 1975

\section{THE NUMBER OF PROPER MINIMAL QUASIVARIETIES OF GROUPOIDS}

\section{A. SHAFAAT}

ABSTRACT. It is shown that if an algebra has more than one element, is freely generated in some variety by one element and has a cancellative endomorphism semigroup then it generates a minimal quasivariety. This is used to construct uncountably many minimal quasivarieties of groupoids that are not varieties.

A quasivariety [1] $\mathcal{K}$ of algebras will be called implicationally complete or minimal if $\mathcal{K}$ has exactly two subquasivarieties, namely, $\mathcal{K}$ itself and the class of all singleton (one element) algebras. By a proper quasivariety we mean a quasivariety which is not a variety.

In the case of semigroups there are countably infinitely many minimal quasivarieties, only one of which is proper [3]. For groupoids in general

Received by the editors February 5, 1973 and, in revised form, January 28, 1974. AMS (MOS) subject classifications (1970). Primary 08A15.

Key words and phrases. Implicationally complete or minimal quasivarieties, (universal) algebras, groupoids, free monogenic groupoid, endomorphism semigroup, cancellative, identity, implication, proper quasivariety, fully invariant congruences, words. 